artery in one-stage should not be done. All other patients showed good recoveries.

Four cases of intracranial arteriovenous malformation were operated on, but the total extirpation of angiomatous portion was very difficult. Hazards were met with chiefly in finding out afferent arteria. Serial angiography may be indispensable to demonstrate afferent and efferent vessels precisely.

Non-traumatic intracerebral hematoma was found unexpectedly in 3 cases during operation under the diagnosis of brain tumor. This experience suggests that if the angiography in early stage following the stroke becomes more common, more patients of intracranal bleeding can be favored with surgery.

\title{
S35. Surgery of Cerebral Aneurysm and Arteriovenous Malformation
}

\author{
Hiroshi FukaI, Keizo Kawakami and Hiraki Tomita \\ Dept. of Neurosurgery, Niigata University School of Medicine
}

We have had 57 cases of cerebral aneurysm and 31 cases of arteriovenous malformation in our clinic, most of which were encountered for these five years. On cerebral angiography which is very important for diagnosis vasodilators are administered and bilateral carotid angiographies are routinely performed because occasionally multiple aneurysms are present ( $9 \%$ in our clinic). To prevent postoperative complications Matas' test and angiography of the non-affected side should be performed to examine development of collaterals. When the neck of aneurysm is not visualized adequately in routine A-P and lateral views another projection is required. Most of aneurysms situated near the anterior half of the circle of Willis are usually visualized only by supra- or infraorbital oblique view. Serial angiography is usually not necessary.

Preoperative mortality rate of aneurysm is $20 \%$. Determination to choose radical operation (neck clipping or trapping) or palliative method (wrapping, removal of hematoma, exploratory craniotomy and ligation of the carotid artery) depends upon frequency of attack, general condition, shape, size, and localization of aneurysm. Radical operation was performed with success in $60 \%$ of craniotomy cases but $15 \%$ succumbed to operation death. On the other hand no death was resulted from palliative operation; esp. in removal of hematoma and ligation of the carotid artery long follow-up study shows remarkable results and these methods should be re-evaluated. In craniotomy cases Fluothane anesthesia is used, urea is administered to reduce brain volume and hypothermia is used only in difficult cases with aneurysms of the anterior communicating 
artery and so on. On rupture of the aneurysm during operation temporary trapping with Mayfield's clip is done, clipping or plastic adhesive agent is applied to check hemorrhage and permanent trapping should be avoided.

In arteriovenous malformation prognosis for useful life is satisfactory by medical or surgical treatment and surgical indication should be determined prudently. Before operation both afferent and efferent vessels are visualized by serial angiography. Extirpation is the most desirable method but is not always feasible. In such cases intracranial ligation of the vessels is carried out. We devised a new method, piloinsertion, in which a few filaments of horse tail were inserted into the malformation through several vessels. This method seems to precipitate thrombus formation, to reduce size of malformation and to minimize opportunity of re-bleeding. More detailed discussion on this method will be followed later.

\title{
S36. Experiences with the Surgical Treatment of Cerebrovascular Diseases
}

\author{
Hajime Handa, Kyozo Ando, Satoshi Shimizu \\ Kouzo Yoshida and Yoshinari KamiJyo \\ Ist Surgical Division, Kyoto University Medical School
}

We have treated 197 patients with cerebrovascular disease in the past 8 years. These included 83 cases of aneurysm, 70 of arteriovenous malformation, 14 of hypertensive hemorrhage and 30 of carotico-vertebral-cerebral stenosis. Based on these experiences, the indication, the time, the procedures of surgery and their prognosis have been reported.

1) Aneurysms of the internal carotid arteries (38 cases: carotid ligation 20 , direct attack 10 , no surgery 8 ). Follow-up study of 3 months to 8 years that the mortality rate with or without surgery in this group is generally low, especially in cases with aneurysm of intracavernous portion. Danger of recurrent hemorrhage is usually expected to occur in cases of aneurysms either at the junction of the posterior communicating artery or at the internal carotid bifurcation, especially in the presence of unconsciousness of long duration, severe headache, and hemiparesis, even transient, etc. In such cases, immediate surgery, especially direct attack is indicated. Carotid ligation was as a rule performed with the use of measurement of the distal intraarterial pressure under local anesthesia. Intraarterial pressure indicates that half of patients shows forward flow direction from external carotid to internal carotid artery after temporary occlusion of the common carotid artery, and half retrograde flow direction. In the former group, if 